\title{
Safety and Effectiveness of Natalizumab: First Report of Interim Results of Post-Marketing Surveillance in Japan
}

\author{
Takahiko Saida - Kazumasa Yokoyama - Ryusuke Sato - Haruki Makioka • \\ Yukihiko Iizuka · Masakazu Hase · Yan Ling · Shinichi Torii
}

Received: May 15, 2017 / Published online: November 8, 2017

(C) The Author(s) 2017. This article is an open access publication

\begin{abstract}
Introduction: Natalizumab, a humanized anti- $\alpha 4$ integrin monoclonal antibody, received marketing approval in Japan in 2014 for the treatment of multiple sclerosis (MS). Because the previous large-scale clinical trials of natalizumab were mainly conducted in Europe and North American countries, and data in patients with MS from Japan were limited, we conducted an all-case post-marketing surveillance of natalizumab-treated MS patients from Japan to investigate the safety and effectiveness of natalizumab in a real-world clinical setting in Japan. Here, we report the results of an interim analysis.
\end{abstract}

Methods: During the observation period of 2 years, all patients who were treated with natalizumab subsequent to its approval in Japan

Enhanced content To view enhanced content for this article go to http://www.medengine.com/Redeem/FBC CF06071BA3F07.

T. Saida $(\bowtie)$

Kansai Multiple Sclerosis Center, Kyoto Min-iren

Central Hospital, Kyoto, Japan

e-mail: saida_takahiko@maia.eonet.ne.jp

K. Yokoyama

Department of Neurology, Juntendo University

School of Medicine, Tokyo, Japan

R. Sato · H. Makioka - Y. Iizuka · M. Hase · Y. Ling ·

S. Torii

Biogen Japan Ltd., Tokyo, Japan were followed. The effectiveness of natalizumab was assessed by examining the changes in expanded disability status scale (EDSS) score and annualized relapse rate (ARR) from baseline. Safety was assessed by analyzing the incidence of adverse drug reactions (ADRs).

Results: The safety analysis included 106 patients (mean age 39.3 years; women 62.3\%) whose data were collected until the data lock point (February 7, 2016). The effectiveness analysis included 75 patients. The majority of patients had relapsing-remitting MS (93/106 patients; $87.7 \%)$. The mean length of treatment exposure in the present study was 6.6 months. During the 2-year observation period, no significant change in the EDSS was observed, while the ARR decreased significantly from baseline (72.9\% reduction, $p=0.001$ ). ADRs and serious ADRs were observed in $11.3 \%$ and $3.8 \%$ of patients, respectively; however, no new safety concerns were detected. No patient had progressive multifocal leukoencephalopathy (PML) during the present study period.

Conclusion: The safety and effectiveness of natalizumab were confirmed in Japanese patients with MS in clinical practice. Nevertheless, potential risks including PML require continuous, careful observation.

Funding: Biogen Japan Ltd (Tokyo, Japan).

Keywords: Japan; Multiple sclerosis; Natalizumab; Post-marketing surveillance 


\section{INTRODUCTION}

Natalizumab is a humanized anti- $\alpha 4$ integrin monoclonal antibody approved and marketed in more than 65 countries for the treatment of multiple sclerosis (MS). Clinical trials of natalizumab began in 1995, and its safety and clinical efficacy in the treatment of MS were confirmed in two international phase 3 studies: the AFFIRM study and the SENTINEL study [1-3]. The AFFIRM study [1], which was conducted in Europe, North America, Australia, and New Zealand, investigated the safety and efficacy of natalizumab monotherapy compared with placebo in patients with relapsing-remitting MS (RRMS). The study showed that natalizumab monotherapy reduced the risk of 6-month confirmed disability worsening as assessed by the expanded disability status scale (EDSS); the number of new or enlarging hyperintense lesions detected by T2-weighted magnetic resonance imaging (MRI); the annualized relapse rate (ARR) after 1 year; and the number of lesions as detected by gadolinium-enhanced MRI. Adverse events (AEs) included fatigue, allergic reaction, pharyngitis, sinus congestion, and peripheral edema.

To investigate the safety and effectiveness of long-term treatment with natalizumab under actual clinical conditions, an observational study, the Tysabri Observational Program (TOP), is being conducted in 17 countries throughout Europe, North America, and South America [4]. In an interim analysis of the TOP study at 5 years of the total 10-year observational period, ARR and EDSS remained low for 5 years after treatment with natalizumab and no new safety issues were observed. However, progressive multifocal leukoencephalopathy (PML) was reported as a serious adverse event in 18 patients $(0.4 \%)$ who had been treated with natalizumab for $\geq 2$ years [4].

PML has been identified as a safety issue in treatment with natalizumab [5-8]. The presence of anti-John Cunningham virus (JCV) antibodies [9], prior treatment with an immunosuppressant (e.g., mitoxantrone, azathioprine, methotrexate, cyclophosphamide, mycophenolate mofetil), and longer treatment duration, especially beyond 2 years, have been identified as risk factors for PML. Patients with these risk factors, as well as those without a history of immunosuppressant use but with a high anti-JCV antibody index and a history of long-term use of natalizumab, have been reported to be at higher risk [10, 11]. Currently, available evidence suggests that the risk of PML is low at an anti-JCV antibody index $\leq 0.9$, whereas it increases substantially at $>1.5$ for patients who have been treated with natalizumab for longer than 2 years.

Epidemiological studies have suggested that the features of MS vary depending on the genetic background of the patients' ethnicity [12]. The Japanese population is genetically homogeneous and geographically isolated. From that point of view, the phase 2 study of natalizumab that enrolled RRMS patients in Japan showed that natalizumab reduced EDSS and ARR with no safety concerns [13, 14]. Consequently, natalizumab was approved in 2014 with indications for prevention of relapse and suppression of disability worsening in MS patients. However, because there were insufficient efficacy and safety data for Japanese patients, this approval was made on the condition that post-marketing surveillance would be conducted for all patients taking natalizumab to evaluate its effectiveness and safety under real-world clinical conditions. There is currently no publication containing real-world data from a substantial number of Japanese patients. This study includes data from an all-case surveillance that has a large number of cases from the clinical setting. Confirmation of its safety and effectiveness in the early phase promotes the appropriate use of natalizumab. Furthermore, this study shows that Japanese MS is genetically different from that of Western populations; for example, we found that DRB ${ }^{*} 0405$ is the highest MS risk allele $[15,16]$. This study is ongoing. Here, we report the results of an interim analysis of data collected until the data lock point at 1 year and 8 months from the start of surveillance. 


\section{METHODS}

The present study was conducted by Biogen Japan, Ltd (Tokyo, Japan) in accordance with the Declaration of Helsinki and the Good Post-marketing Study Practice in Japan. The study included all MS patients who had received treatment with natalizumab (Tysabri ${ }^{\circledR}$ ) since its launch, using a central registration system. Patients who had participated in the phase 2 extension study of natalizumab were also enrolled in the present study at the time they switched from the preapproval form to the marketed form of the drug. The target number of patients was 400 . The registration and study periods were from June 4, 2014 to the completion of 2 years of observation of the last patient to be enrolled. Data as of February 7, 2016 (data lock point) were aggregated and used for analysis. The standard observation period of this study was 2 years, except in cases where the treatment was discontinued. If treatment was discontinued, patients were followed up for 6 months after discontinuation. Data were collected through case report forms recorded by the attending physicians during routine clinical practice visits.

The major investigated items included patient characteristics, relapse rates, changes in EDSS, and the incidence rates of AEs and adverse drug reactions (ADRs). ADRs were defined as AEs for which the causal relationship with natalizumab could not be ruled out. Additionally, the important investigated items for safety were specified as anaphylaxis, PML, infections other than PML, immune reconstitution inflammatory syndrome (IRIS), liver damage, anti-natalizumab antibody production, and JCV antibodies. Table 1 shows the study schedule.

Changes in EDSS over time from baseline were analyzed using the Wilcoxon signed-rank test. Relapse was evaluated by calculating ARR. Changes in ARR with 95\% confidence intervals (CI) at each time point from baseline were

Table 1 Study schedule

\begin{tabular}{|c|c|c|}
\hline Survey criteria & $\begin{array}{l}\text { Questionnaire form } \\
\text { for } 1 \text { year }(0-12 \mathrm{M})\end{array}$ & $\begin{array}{l}\text { Questionnaire form } \\
\text { for } 2 \text { years }(12-24 \mathrm{M})\end{array}$ \\
\hline Patient background & $\bigcirc$ & - \\
\hline Primary disease & $\bigcirc$ & - \\
\hline Condition of administration & $\bigcirc$ & $\bigcirc$ \\
\hline Discontinuation/dropout & $\bigcirc$ & $\bigcirc$ \\
\hline Previous medication/treatment & $\bigcirc$ & - \\
\hline Concomitant drug/treatment & $\bigcirc$ & $\bigcirc$ \\
\hline Anti-JCV antibody & $0,6,12 \mathrm{M}$ & $18,24 \mathrm{M}$ \\
\hline Anti-natalizumab antibody & $\bigcirc$ & O \\
\hline Anti-AQP4 antibody & O & O \\
\hline Clinical course & $\bigcirc$ & O \\
\hline Relapse & O & $\bigcirc$ \\
\hline EDSS $^{\mathrm{a}}$ & $0,3,6,9,12 \mathrm{M}$ & $15,18,21,24 \mathrm{M}$ \\
\hline Adverse event & $\bigcirc$ & $\bigcirc$ \\
\hline Laboratory testing & $\bigcirc$ & $\bigcirc$ \\
\hline
\end{tabular}

$M$ months, JCV John Cunningham virus, AQP4 aquaporin-4, EDSS expanded disability status scale

a Since EDSS was measured in a real-world clinical setting, the last evaluated score was used for analysis 
analyzed using a negative binomial regression model. For each analysis, $p<0.05$ was considered statistically significant. SAS release 9.3 (SAS Institute, Cary, NC, USA) was used for data analysis. AEs were encoded using the Medical Dictionary for Regulatory Activities Terminology (MedDRA)/J version 18.1 and classified according to their preferred terms.

\section{RESULTS}

\section{Patients}

As of the data lock point (February 7, 2016), a total of 170 Japanese MS patients who received natalizumab were registered. Data were collected from 169 patients after excluding 1 patient who did not take natalizumab. Of them, 106 patients whose data were locked were included in the safety analysis. For the remaining patients, data are expected to be locked after confirmation of data adequacy. A total of 75 patients were included in the effectiveness analysis after excluding 30 patients whose effectiveness data were unlocked and 1 patient whose efficacy data were not collected (Fig. 1). The safety analysis population included 33 patients from the phase 2 study in Japan who continuously received treatment with natalizumab.

Table 2 shows the patient baseline characteristics of the safety analysis population consisting of patients from the phase 2 study $(n=33)$ and natalizumab-naïve patients $(n=73)$, as well as all patients. Women accounted for $62.3 \%$; mean age was 39.3 years; mean body weight was $55.3 \mathrm{~kg}$. There was 1 patient with a low body weight $<40 \mathrm{~kg}$. Natalizumab was used for the treatment of MS in all 106 patients. The majority of the patients had RRMS (87.7\%), and there were no patients with primary progressive MS. Most baseline characteristics were similar between patients from the phase 2 study and natalizumab-naive patients except for the percentage of patients who received prior MS therapy and the number of relapses in the past 1 year. All 106 patients were tested for anti-JCV antibodies, and 66 patients $(62.3 \%)$ were positive. Of the 99 patients who were tested for anti-aquaporin-4 (AQP4) antibodies, none were found to be positive.

\section{Treatment Exposure}

The mean length of treatment exposure in the present study was 6.6 months (for those who had continuously participated in the clinical

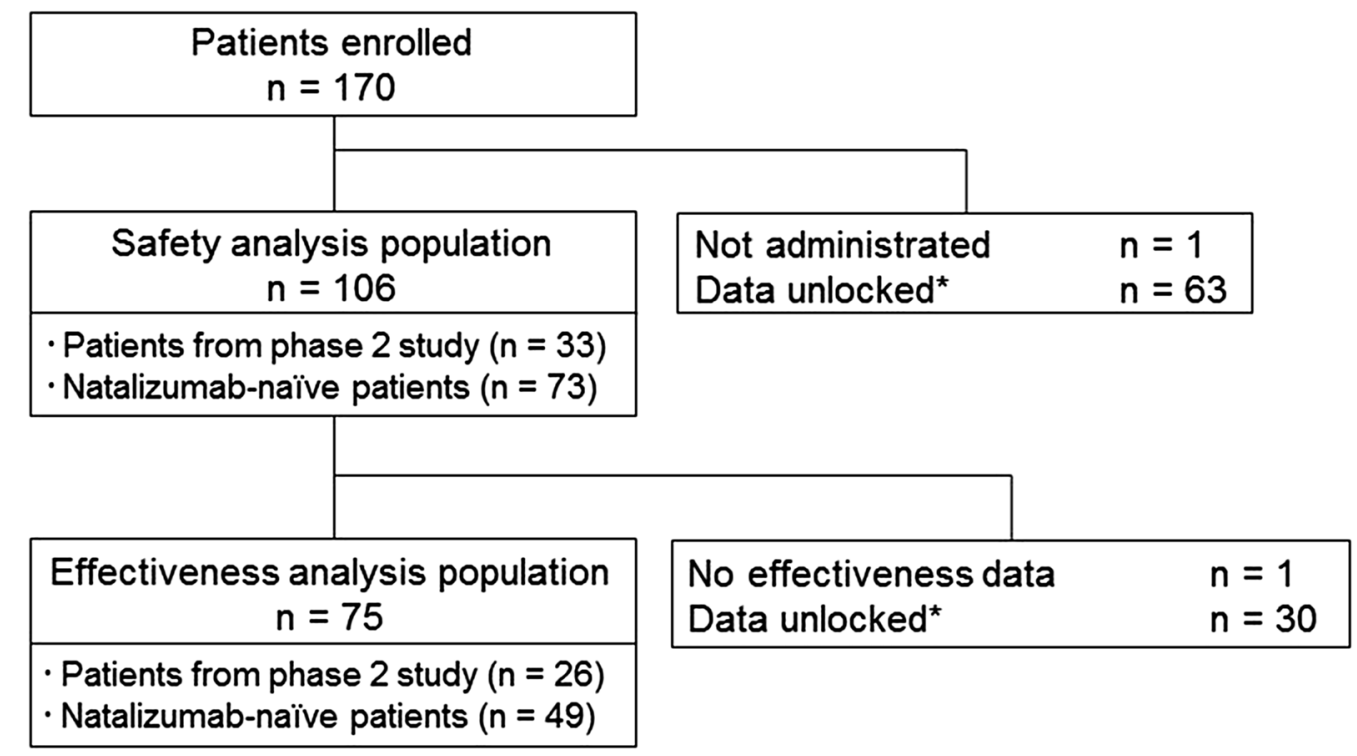

Fig. 1 Patient disposition. Asterisk: as of February 7, 2016 
Table 2 Baseline characteristics

\begin{tabular}{|c|c|c|c|}
\hline Items & All & $\begin{array}{l}\text { Patients from phase } \\
2 \text { study }\end{array}$ & $\begin{array}{l}\text { Natalizumab-naïve } \\
\text { patients }\end{array}$ \\
\hline Total number of patients, $n(\%)$ & 106 & 33 & 73 \\
\hline Female, $n(\%)$ & $66(62.3)$ & $21(63.6)$ & $45(61.6)$ \\
\hline \multicolumn{4}{|l|}{ Inpatient/outpatient status, $n(\%)$} \\
\hline Outpatient & $94(88.7)$ & $33(100.0)$ & $61(83.6)$ \\
\hline Inpatient & $7(6.6)$ & $0(0.0)$ & $7(9.6)$ \\
\hline Inpatient/outpatient & $5(4.7)$ & $0(0.0)$ & $5(6.8)$ \\
\hline \multicolumn{4}{|l|}{ Age (years) ${ }^{\mathrm{a}}$} \\
\hline Mean \pm SD & $39.3 \pm 10.8$ & $40.2 \pm 8.5$ & $38.8 \pm 11.7$ \\
\hline Median $[\min , \max ]$ & $39.0[15,78]$ & $39.5[26,59]$ & $39.0[15,78]$ \\
\hline$<15$ & $0(0.0)$ & $0(0.0)$ & $0(0.0)$ \\
\hline$\geq 15$ and $<65$ & $94(88.7)$ & $32(97.0)$ & $62(84.9)$ \\
\hline$\geq 65$ & $2(1.9)$ & $0(0.0)$ & $2(2.7)$ \\
\hline \multicolumn{4}{|l|}{ Body weight $(\mathrm{kg})^{\mathrm{b}}$} \\
\hline Mean $\pm S D$ & $55.3 \pm 10.9$ & $56.8 \pm 11.0$ & $54.7 \pm 9.7$ \\
\hline Median $[\min , \max ]$ & $53.5[39.9,86.0]$ & $54.5[43.0,86.0]$ & $52.0[39.9,74.0]$ \\
\hline \multicolumn{4}{|l|}{ MS type ${ }^{c}, n(\%)$} \\
\hline Clinically isolated syndrome & $0(0.0)$ & $0(0.0)$ & $0(0.0)$ \\
\hline Relapsing-remitting type & $93(87.7)$ & $29(87.9)$ & $64(87.7)$ \\
\hline Secondary progressive type & $13(12.3)$ & $4(12.1)$ & $9(12.3)$ \\
\hline Primary progressive type & $0(0.0)$ & $0(0.0)$ & $0(0.0)$ \\
\hline \multicolumn{4}{|l|}{ Years from onset of MS (years) ${ }^{\mathrm{d}}$} \\
\hline Mean $\pm S D$ & $10.0 \pm 6.6$ & $10.8 \pm 5.9$ & $9.7 \pm 6.8$ \\
\hline Median $[\min , \max ]$ & $8.5[0.5,33.4]$ & $8.6[3.2,26.3]$ & $8.1[0.5,33.4]$ \\
\hline \multicolumn{4}{|l|}{ Prior MS therapy, $n(\%)$} \\
\hline DMT-naïve & $20(18.9)$ & $7(21.2)$ & $13(17.8)$ \\
\hline IFN only & $34(32.1)$ & $21(63.6)$ & $13(17.8)$ \\
\hline Fingolimod only & $19(17.9)$ & $0(0.0)$ & $19(26.0)$ \\
\hline Switched between IFN and fingolimod & $33(31.1)$ & $5(15.2)$ & $28(38.4)$ \\
\hline \multicolumn{4}{|l|}{ Number of relapses in past 1 year, $n(\%)$} \\
\hline 0 & $58(54.7)$ & $26(78.8)$ & $32(43.8)$ \\
\hline $1^{\mathrm{e}}$ & $25(23.6)$ & $3(9.1)$ & $22(30.1)$ \\
\hline $2^{e}$ & $13(12.3)$ & $2(6.1)$ & $11(15.1)$ \\
\hline
\end{tabular}


Table 2 continued

\begin{tabular}{llll}
\hline Items & All & $\begin{array}{l}\text { Patients from phase } \\
\text { 2 study }\end{array}$ & $\begin{array}{l}\text { Natalizumab-naïve } \\
\text { patients }\end{array}$ \\
\hline$\geq 3^{\mathrm{e}}$ & $7(6.6)$ & $1(3.0)$ & $6(8.2)$ \\
Unknown & $3(2.8)$ & $1(3.0)$ & $2(2.7)$ \\
Mean $\pm \mathrm{SD}^{\mathrm{e}}$ & $0.7 \pm 1.0$ & $0.3 \pm 0.9$ & $0.9 \pm 1.1$ \\
Median [min, max $]^{\mathrm{e}}$ & $0.0[0,4]$ & $0.0[0,4]$ & $1.0[0,4]$ \\
EDSS score & & $2.53 \pm 1.97$ & $3.05 \pm 2.47$ \\
Mean $\pm \mathrm{SD}$ & $2.89 \pm 2.33$ & $2.00[0.0,7.0]$ & $2.50[0.0,8.5]$ \\
Median [min, $\max ]$ & $2.00[0.0,8.5]$ &
\end{tabular}

$M S$ multiple sclerosis, DMT disease-modifying therapy, EDSS expanded disability status scale, $S D$ standard deviation

a $n=96$ overall; patients from phase 2 study, $n=32$; natalizumab-naïve patients, $n=64$

b $n=93$ overall; patients from phase 2 study, $n=28$; natalizumab-naïve patients, $n=65$

c MS type was diagnosed by physicians

d $n=105$ overall; natalizumab-naïve patients, $n=72$

e Except for unknown patients $(n=103$ overall; patients from the phase 2 study, $n=32$; natalizumab-naïve patients, $n=71)$

f $n=104$ overall; patients from the phase 2 study, $n=32$; natalizumab-naïve patients, $n=72$

trials for market approval of natalizumab, only the treatment period of the present study was counted). No patients received treatment for $\geq 18$ months (Table 3 ). A total of 27 patients (25.5\%) discontinued natalizumab (Table 4). The reasons for treatment discontinuation were insufficient effect and worsening of MS in six patients (5.7\%), AEs in five patients (4.7\%), patient request in two patients $(1.9 \%)$, no hospital visit in one patient $(0.9 \%)$, and pregnancy in one patient $(0.9 \%)$. Other reasons (14 patients, $13.2 \%$ ) included concerns about the risk of PML in ten patients (9.4\%). Eleven patients $(10.4 \%)$ were tested for anti-natalizumab antibodies; three patients (2.8\%) were positive for anti-natalizumab antibodies. There was no report of relapse in with patients who were positive for anti-natalizumab antibodies.

\section{Effectiveness}

Effectiveness was evaluated via changes in EDSS score and ARR from baseline. The effectiveness analysis population included patients who had continued treatment from the phase 2 extension study; in those patients, EDSS and ARR had improved at the baseline of the present study. Therefore, to evaluate the effectiveness in natalizumab-naïve patients, in addition to the original planned effectiveness analysis of the total population, we performed additional analyses of EDSS and ARR after excluding patients from the phase 2 study.

EDSS data were collected from 60 patients. In the total effectiveness analysis population, there was no significant difference in EDSS

Table 3 Duration of natalizumab exposure

\begin{tabular}{ll}
\hline Total (months) & \\
\hline Mean \pm SD & $\mathbf{6 . 6} \pm \mathbf{5 . 5}$ \\
Median [min, max] & $\mathbf{9 . 5}[\mathbf{0 . 0}, \mathbf{1 6 . 7}]$ \\
\hline$<6$ months, $n$ (\%) & $49(46.2)$ \\
$\geq 6$ and $<12$ months, $n(\%)$ & $41(38.7)$ \\
$\geq 12$ and $<18$ months, $n(\%)$ & $16(15.1)$ \\
$\geq 18$ and $<24$ months, $n(\%)$ & $0(0.0)$ \\
$\geq 24$ months, $n(\%)$ & $0(0.0)$ \\
\hline
\end{tabular}

$S D$ standard deviation 


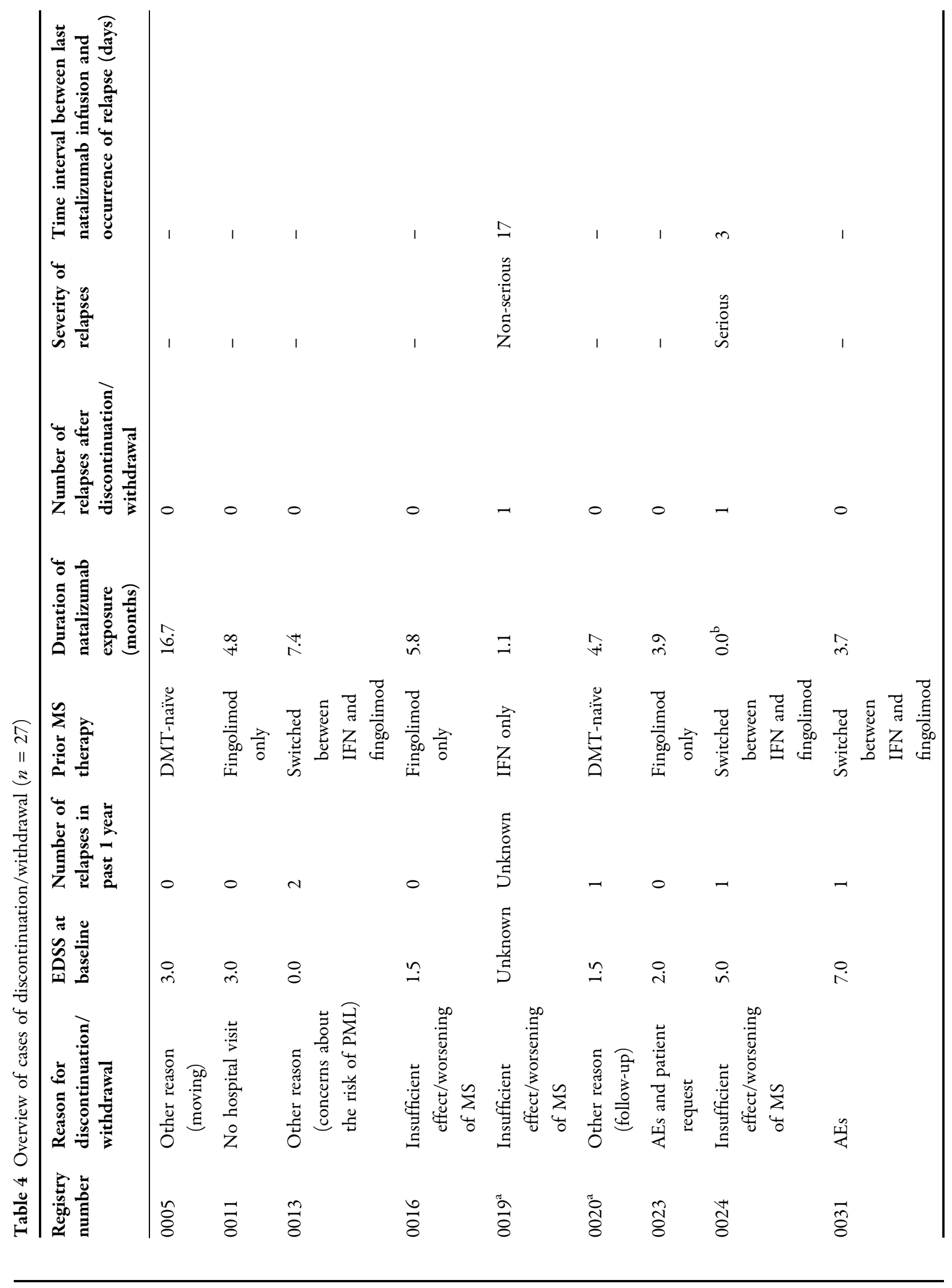




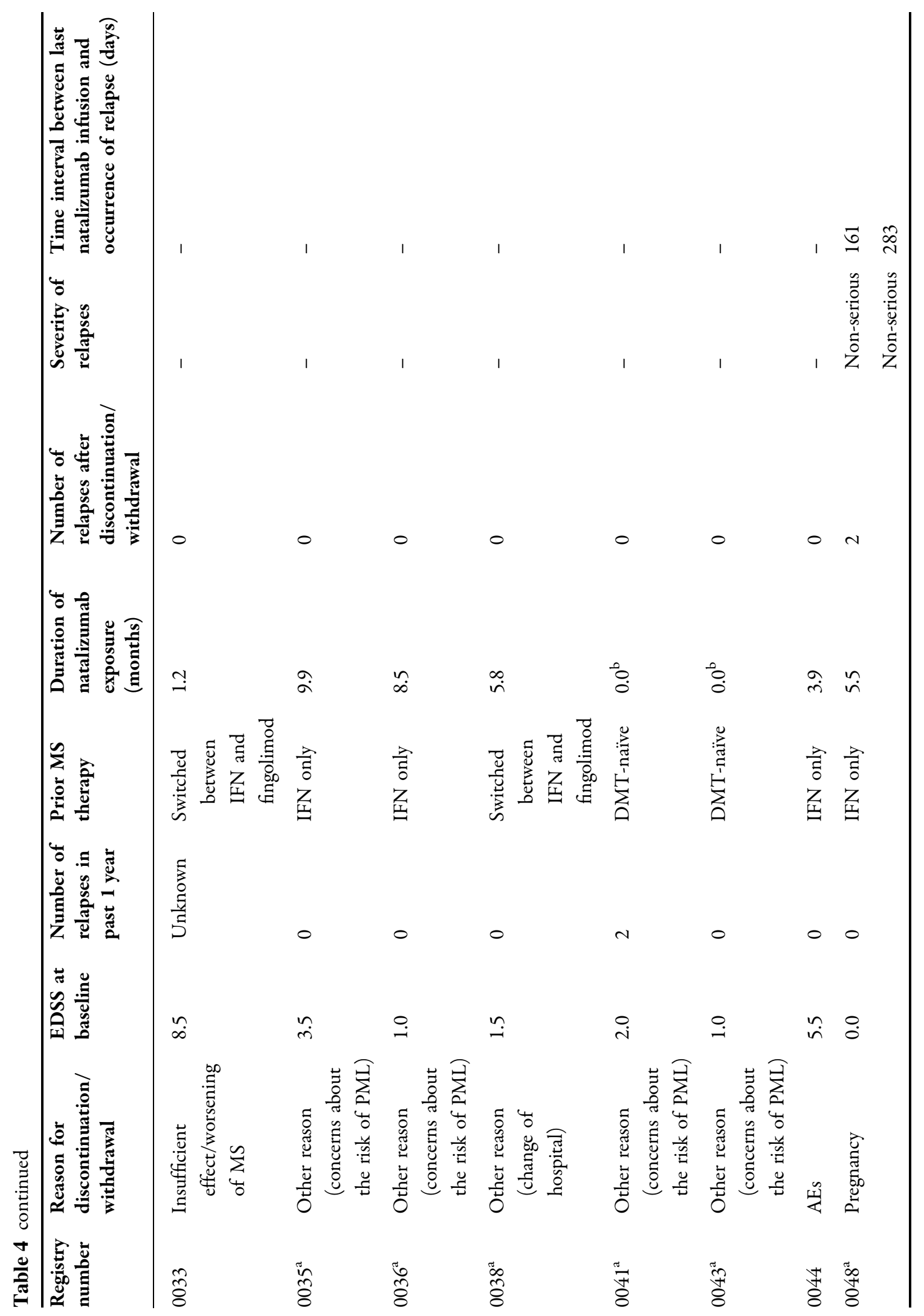




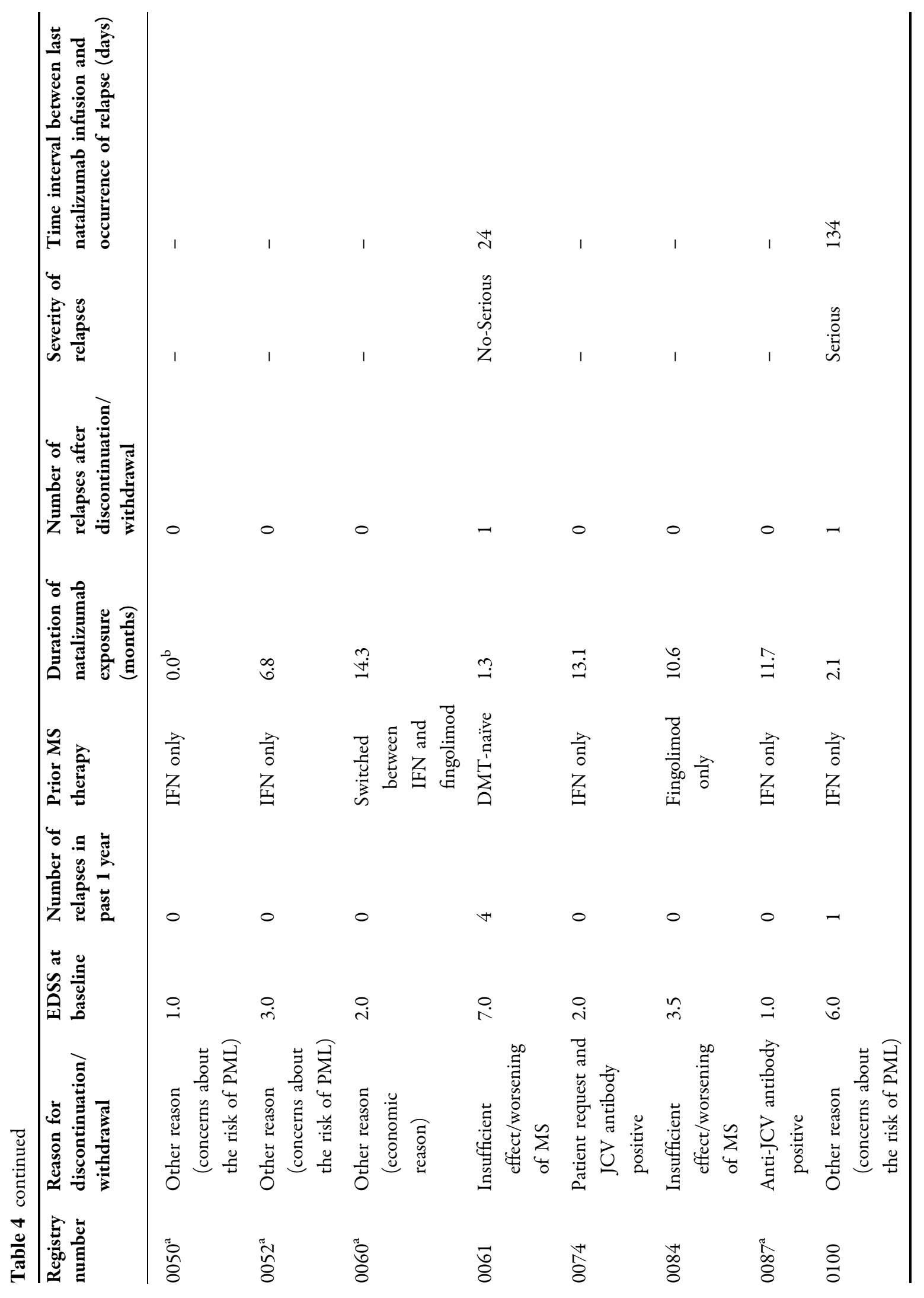




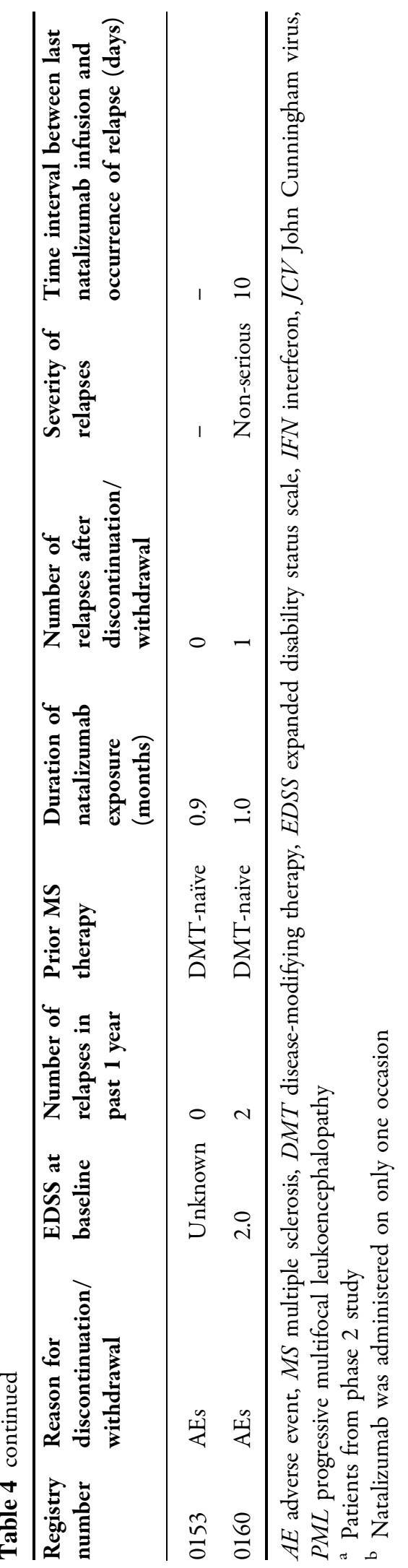

between baseline and the last observation (Fig. 2, $p=0.290$ ). After excluding patients from the phase 2 study $(n=22)$, the EDSS change between baseline (2.59) and the last observation (2.50) was $0.09(p=0.349)$. The EDSS change in patients from the phase 2 study was 0.07 (2.27 to $2.20, p=1.000)$. Improved and worsened EDSS were defined as a decrease of more than 1 and an increase of more than 0.5 from baseline, respectively. The proportions of the cases that were stable, improved, or worsened were $82 \%$, $10 \%$, and $8 \%$, respectively. There were nine patients who had baseline EDSS > 5.0; the mean EDSS in these patients at baseline and at the last evaluation was 6.50 and 6.28 , respectively.

ARR data were collected from 74 patients. In the total effectiveness analysis population, there was a significant improvement in ARR; the ARR of patients at 1 year before treatment and at the end of treatment were 0.59 (95\% CI $0.38-0.83)$ and 0.16 (95\% CI $0.09-0.31)$, respectively ( $p=0.001$ ) (Fig. 3). 21.6\% (16/74) of the patients were treated for more than 1 year and the ARR of the group was 0.38 before treatment and 0.06 at the end of treatment $(p=0.092)$. The ARR of patients from the phase 2 study $(n=26)$ was 0.23 (95\% CI $0.10-0.66)$ before treatment and 0.14 (95\% CI 0.07-0.42) at the end of treatment $(p=0.357)$. After excluding patients from the phase 2 study $(n=48)$, ARRs were 0.79 (95\% CI $0.50-1.12)$ and 0.18 (95\% CI $0.09-0.38)$, respectively $(p=0.001)$. Forty-two patients were on another DMT before registration, and their ARRs before and at the end of treatment were 0.71 (95\% CI 0.45-1.07) and 0.14 (95\% CI $0.06-0.32)$, respectively $(p=0.003)$.

\section{Safety}

Of the 106 patients included in the safety analysis, ADRs were observed in 12 patients $(11.3 \%)$, of whom four patients $(3.8 \%)$ had serious ADRs (Table 5). Major ADRs included hepatic function abnormal and pyrexia, and no new ADRs were noted. Major serious ADRs were MS relapse, neurosis, and suicide attempt. No patient had PML during the observation period. ADRs that caused treatment interruption or 


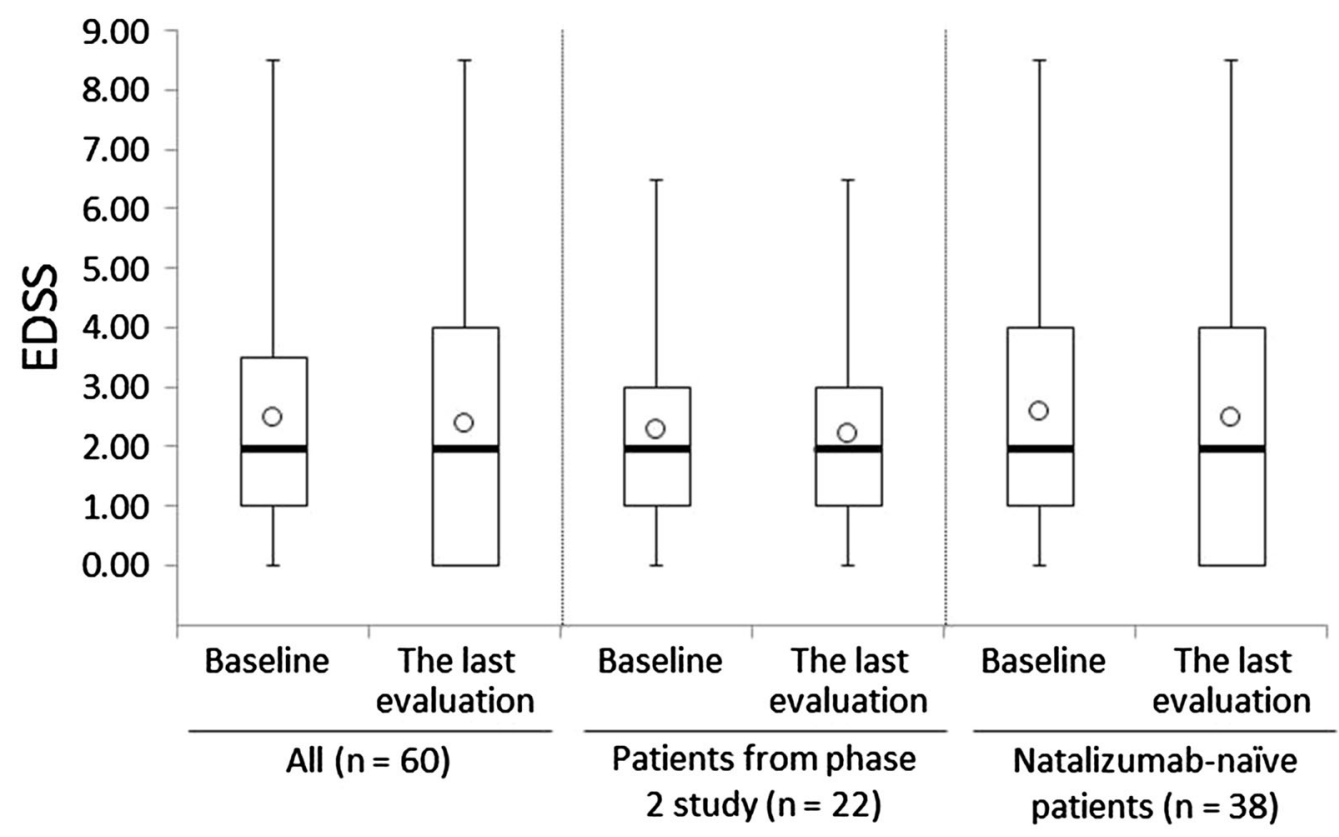

Fig. 2 Box plot of the expanded disability status scale (EDSS) scores in the total effectiveness population. The heavy horizontal lines show the median, the boxes show the

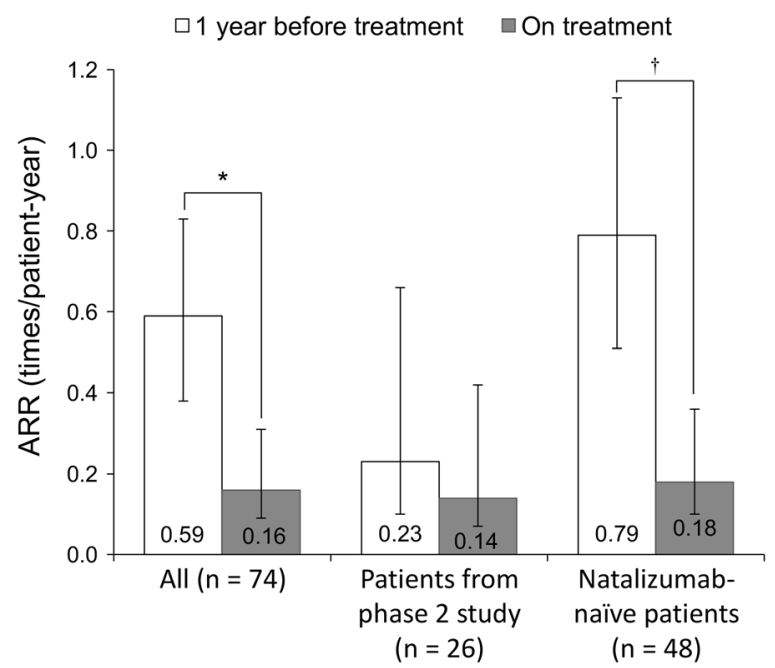

Fig. 3 Annualized relapse rate (ARR) at 1 year before and while on treatment with natalizumab in the total effectiveness population. White bar shows ARR 1 year before treatment, gray bar shows ARR while on treatment. The error bars indicate $95 \%$ confidence intervals. ${ }^{*} p=0.001,{ }^{\dagger}$ $p<0.001$ by negative binominal regression

discontinuation were observed in seven patients (nine events), including two events of MS relapse and one event each of eczema, tremor, interquartile range, the error bars show the maximum and minimum values, and the open circles show the mean value

suicide attempt, chills, resting tremor, back pain, and pruritus generalised. One patient required a reduced dose of natalizumab because of a non-serious ADR: hepatic function abnormal. After discontinuation of natalizumab, there were relapses (seven relapses in six patients out of 28 patients) within 6 months from discontinuation (Table 4). The length of treatment exposure of four patients was less than 1.5 months.

\section{DISCUSSION}

This is the first report of the enrollment of a large number of patients to evaluate the safety and effectiveness of natalizumab in a real-world clinical practice setting in Japan. This report is based on the results of an interim analysis at 1 year and 8 months of the all-case post-marketing surveillance with a 2-year standard observation period. Regarding effectiveness, there was no worsening in the EDSS, and ARR significantly decreased. The safety profiles were consistent with those reported previously. 
Table 5 Adverse drug reactions (ADRs) and serious ADRs $(n=106)$

\begin{tabular}{|c|c|c|}
\hline \multirow[t]{2}{*}{ Preferred term } & \multicolumn{2}{|c|}{$\begin{array}{l}\text { Number of events, } \\
n(\%)\end{array}$} \\
\hline & Serious & Non-serious \\
\hline Total (patients/patients) & $12(11.3)$ & \\
\hline Total (case/patients) & $16(15.1)$ & \\
\hline $\begin{array}{l}\text { Total serious ADRs } \\
\text { (patients/patients) }\end{array}$ & $4(3.8)$ & \\
\hline Hepatobiliary disorders & $0(0.0)$ & $3(2.8)$ \\
\hline Alcoholic liver disease & $0(0.0)$ & $1(0.9)$ \\
\hline Hepatic function abnormal & $0(0.0)$ & $2(1.9)$ \\
\hline $\begin{array}{l}\text { Skin and subcutaneous tissue } \\
\text { disorders }\end{array}$ & $0(0.0)$ & $2(1.9)$ \\
\hline Eczema & $0(0.0)$ & $1(0.9)$ \\
\hline Pruritus generalised & $0(0.0)$ & $1(0.9)$ \\
\hline Nervous system disorders & $2(1.9)$ & $2(1.9)$ \\
\hline MS relapse & $2(1.9)$ & $0(0.0)$ \\
\hline Tremor & $0(0.0)$ & $1(0.9)$ \\
\hline Resting tremor & $0(0.0)$ & $1(0.9)$ \\
\hline Psychiatric disorders & $2(1.9)$ & $0(0.0)$ \\
\hline Neurosis & $1(0.9)$ & $0(0.0)$ \\
\hline Suicide attempt ${ }^{\mathrm{a}}$ & $1(0.9)$ & $0(0.0)$ \\
\hline $\begin{array}{l}\text { General disorders and } \\
\text { administration site conditions }\end{array}$ & $0(0.0)$ & $3(2.8)$ \\
\hline Pyrexia & $0(0.0)$ & $2(1.9)$ \\
\hline Chills & $0(0.0)$ & $1(0.9)$ \\
\hline Infections and infestations & $0(0.0)$ & $1(0.9)$ \\
\hline Herpes zoster & $0(0.0)$ & $1(0.9)$ \\
\hline $\begin{array}{l}\text { Musculoskeletal and connective } \\
\text { tissue disorders }\end{array}$ & $0(0.0)$ & $1(0.9)$ \\
\hline Back pain & $0(0.0)$ & $1(0.9)$ \\
\hline
\end{tabular}

$M S$ multiple sclerosis

${ }^{a}$ We received additional information that was unlocked as of February 7, as follows: completed suicide (outcome: death)
In the present study, there were no significant changes in mean EDSS from baseline throughout the observation period, which indicated sustained suppression of disease worsening. There was a statistically significant reduction in ARR from 0.59 to 0.16 , and $86.5 \%$ of patients had no relapse. Moreover, in the natalizumab-naïve patients (i.e., the population remaining after excluding patients from the phase 2 study), the reduction in ARR from 0.79 to 0.18 was greater than that seen in the total effectiveness population, and $85.4 \%$ of patients had no relapse. In previous reports, the mean ARR at 1 year after treatment with natalizumab ranged between 0.27 and 0.38 , and the proportion of patients with no relapse was $72-80 \%$; thus, the endpoints concerning ARR in the present study were similar to those reported previously $[1,3]$. In total, 33 patients from the phase 2 study and the extension study $[13,14]$ were enrolled in the present surveillance. Among these patients, the ARRs of patients at 1 year before treatment and at the end of treatment were 0.23 and 0.14 , respectively. Even after 2 years of treatment extension, ARR was reduced by $47.8 \%$. This result shows the long-term effectiveness of natalizumab.

In the major phase 3 studies of natalizumab $[1,3]$, patients with baseline EDSS $>5.0$ were excluded. However, the effectiveness population of the present study included nine patients $(15.3 \%)$ with baseline EDSS $>5.0$. The ongoing TOP observational study also included patients with high EDSS; in its interim report, $29 \%$ of the patients had EDSS of 4.5-9.5, showing that natalizumab is being used in patients covering the full spectrum of disability in real-world clinical practice [4]. The present study showed that EDSS in patients with baseline EDSS $>5.0$ decreased with natalizumab, but not significantly. However, as there were only a small number of patients with high EDSS in the present study, including three with secondary progressive MS, interpretation is difficult. We plan to report the safety and effectiveness results for those patients with high EDSS by conducting stratified analysis when sufficient data from a certain number of patients have been accumulated.

The ratios of neuromyelitis optica (NMO)/ NMO spectrum disorder (NMOSD) to MS are 
higher in Asia than in Western countries, indicating that differentiating between $\mathrm{NMO} /$ NMOSD and MS is a major challenge in Asia. The detection of the AQP4 antibody is crucial for differentiating NMO/NMOSD from MS and promptly initiating immunosuppressive therapy for NMO/NMOSD [17]. There was no patient in whom anti-AQP4 antibodies were detected in this study. Although the detection of the AQP4 antibody has facilitated early distinction between NMO/NMOSD and MS, up to $40 \%$ of patients with NMO/NMOSD remain seronegative for the AQP4 antibody. Recently, autoantibodies against myelin oligodendrocyte glycoprotein (MOG) were reported in the sera of adult patients with the NMO/NMOSD phenotype [18]. A previous study showed that natalizumab was not beneficial for NMO/NMOSD, and that it may even exacerbate the disease during or shortly after therapy [19]. In this study, Japanese physicians seemed to have reached the differential diagnosis correctly. However, to achieve a more accurate differential diagnosis, additional assessment may be required for patients who do not respond to natalizumab.

In the AFFIRM and SENTINEL studies, 4\% and $1.9 \%$ of patients in the natalizumab-treated groups experienced hypersensitivity reactions, respectively. In previous clinical trials, most of the hypersensitivity reactions occurred on the second infusion, and most patients with a hypersensitivity reaction (68\%) had persistent (one positive test, the result of which was reproducible on retesting at least 6 weeks later) antibodies to natalizumab. The incidence of persistent anti-natalizumab antibodies was $6 \%$ in the entire treatment cohort [20]. In the present study, there were three patients $(2.8 \%)$ who were positive for anti-natalizumab antibodies and they were detected on the second infusion. Patients with anti-natalizumab antibodies experienced hypersensitivity reactions and then discontinued natalizumab immediately. The persistence of antibodies in those patients was not examined. Two out of three patients had ADRs including pruritus generalised. In the present study, the discontinuation rate was $25.5 \%$, which seemed higher than that in a report of the 5-year interim results of the large observational TOP study (25.3\%) [4]. Among the various reasons for discontinuation, concern about the risk of PML was much higher among Japanese neurologists while the other reasons were comparable, suggesting that Japanese physicians may be more careful about the risk of PML. This apparent trend needs to be verified over longer observational periods. On the other hand, there is concern about rebound or reactivation of disease activity after natalizumab discontinuation. Regarding this matter, there have been several studies that have examined whether rebound or reactivation occurs after discontinuation, but their findings are controversial $[21,22]$. In the present study, 6 out of 28 patients who discontinued natalizumab had a relapse after discontinuation; however, four of those six patients had discontinued natalizumab after less than 1.5 months of treatment. In addition, information on disease activity obtained by MRI after natalizumab discontinuation is limited in the present study, since MRI imaging data were not collected. In the present study, no ADRs that raised safety concerns were observed. No patient experienced PML during the present study period. However, after the data lock point of the present study, one patient who had experienced PML after natalizumab treatment was reported in September 2016 in Japan. Although there was no occurrence of PML during the present study period, none of the patients had received the treatment for $\geq 18$ months, and the majority $(84.9 \%)$ of the patients received the treatment for $<1$ year. However, there were some patients who continued from the phase 2 study and were exposed to natalizumab for $>2$ years. Continuous, close attention to the risk of PML is required. Currently, identified risk factors for PML associated with natalizumab include anti-JCV antibody seropositivity, a history of immunosuppressant use, and treatment with natalizumab for $\geq 2$ years [11]. Moreover, in patients without prior immunosuppressant exposure, the risk of developing PML increases with the antibody index [23]. Anti-JCV antibody-positive MS patients were reported to comprise $56 \%$ of all patients at baseline in the observational study STRATIFY-1 in the USA [9], between $47 \%$ and $68 \%$ in the European cohort 
study [24], and 63\% in Japanese phase 2 study [13], which are consistent with the results of the present study (62.3\%).

\section{STUDY LIMITATIONS}

The surveillance was designed to represent a real-world clinical setting; consequently, patients were not blinded to treatment and no placebo comparison was used. Since this was the first interim report of the surveillance, there were relatively few patients and the duration of exposure was relatively short.

\section{CONCLUSIONS}

As a result of the interim analysis at 20 months of the 2-year post-marketing surveillance period, the effects of natalizumab on controlling EDSS progression and preventing MS relapse were confirmed under real-world clinical conditions in Japan. The safety profile was similar to that reported previously. The all-case post-marketing surveillance, which was a condition of approval, is still ongoing, and we consider that the results of the present interim report contribute to the proper use of natalizumab in accordance with the package insert.

\section{ACKNOWLEDGEMENTS}

This study and article processing charges were funded by Biogen Japan Ltd (Tokyo, Japan). All authors meet the International Committee of Medical Journal Editors (ICMJE) criteria for authorship for this manuscript, take responsibility for the integrity of the work as a whole, and have given final approval of the version to be published. The authors thank Hirotaka Narumiya at Intellim Corporation (Tokyo, Japan), for statistical analysis assistance. The authors also thank Kokoro Hayashi, Ph.D, of inScience Communications, Springer Healthcare (Tokyo, Japan) for preparing the first draft and subsequent drafts of this manuscript based on input from authors. Biogen Japan Ltd provided funding for this assistance.
Disclosures. T. Saida has received funding from, held board membership of, spoken at scientific meetings for, prepared manuscripts for, and had consulting agreements with Astellas Pharma, Bayer Schering, Biogen Japan, Daiichi Sankyo, Eisai, Kaketsuken, Merck Serono, Mitsubishi Tanabe Pharma, Nihon Pharmaceutical, Novartis Pharma, Ono Pharmaceutical, Sanofi, TDS Japan, and Teijin. K. Yokoyama has received funding from, spoken at scientific meetings for, and prepared manuscripts for Novartis Pharma, Mitsubishi Tanabe Pharma, Astellas Pharma, Biogen Japan, Nihon Pharmaceutical, and Japan Blood Products Organization, research grant for Takeda Research Support and is a member of a laboratory funded by ABIST, Asahi Kasei Medical, Bayer Yakuhin, Biogen Japan, Daiwa, Hydrogen Health Medical Labo, Japan Blood Products Organization, Kenko Kazoku, Melodian, Mitsubishi Tanabe, MiZ, and Nihon Pharmaceutical. R. Sato is an employee and stockholder of Biogen Japan. H. Makioka is an employee and stockholder of Biogen Japan. Y. Iizuka is an employee and stockholder of Biogen Japan. M. Hase is an employee and stockholder of Biogen Japan. Y. Ling is an employee and stockholder of Biogen Japan. S. Torii is an employee and stockholder of Biogen Japan.

Compliance With Ethics Guidelines. This study was conducted as an all-case post-marketing surveillance of natalizumab in accordance with the Declaration of Helsinki and Good Post-Marketing Study Practice in Japan.

Data Availability. The datasets generated and/or analyzed during the current study are not publicly available and are fully owned by Biogen, but are available from HM at haruki.makioka@biogen.com on reasonable request.

Open Access. This article is distributed under the terms of the Creative Commons Attribution-NonCommercial 4.0 International License (http://creativecommons.org/licenses/ by-nc/4.0/), which permits any noncommercial use, distribution, and reproduction in any medium, provided you give appropriate credit to the original author(s) and the source, provide 
a link to the Creative Commons license, and indicate if changes were made.

\section{REFERENCES}

1. Polman $\mathrm{CH}, \mathrm{O}^{\prime}$ Connor PW, Havrdova E, et al. A randomized, placebo-controlled trial of natalizumab for relapsing multiple sclerosis. $\mathrm{N}$ Engl J Med. 2006;354:899-910.

2. Havrdova E, Galetta S, Hutchinson M, et al. Effect of natalizumab on clinical and radiological disease activity in multiple sclerosis: a retrospective analysis of the Natalizumab Safety and Efficacy in Relapsing-Remitting Multiple Sclerosis (AFFIRM) study. Lancet Neurol. 2009;8:254-60.

3. Rudick RA, Stuart WH, Calabresi PA, et al. Natalizumab plus interferon beta-1a for relapsing multiple sclerosis. N Engl J Med. 2006;354:911-23.

4. Butzkueven H, Kappos L, Pellegrini F, et al. Efficacy and safety of natalizumab in multiple sclerosis: interim observational programme results. J Neurol Neurosurg Psychiatry. 2014;85:1190-7.

5. Kleinschmidt-DeMasters BK, Tyler KL. Progressive multifocal leukoencephalopathy complicating treatment with natalizumab and interferon beta-1a for multiple sclerosis. $\mathrm{N}$ Engl J Med. 2005;353:369-74.

6. Van Assche G, Van Ranst M, Sciot R, et al. Progressive multifocal leukoencephalopathy after natalizumab therapy for Crohn's disease. N Engl J Med. 2005;353:362-8.

7. Langer-Gould A, Atlas SW, Green AJ, et al. Progressive multifocal leukoencephalopathy in a patient treated with natalizumab. $\mathrm{N}$ Engl J Med. 2005;353:375-81.

8. Yousry TA, Major EO, Ryschkewitsch C, et al. Evaluation of patients treated with natalizumab for progressive multifocal leukoencephalopathy. N Engl J Med. 2006;354:924-33.

9. Bozic C, Richman S, Plavina $\mathrm{T}$, et al. Anti-John Cunnigham virus antibody prevalence in multiple sclerosis patients: baseline results of STRATIFY-1. Ann Neurol. 2011;70:742-50.

10. Biogen Japan Ltd. Tysabri ${ }^{\circledR}$ package insert (ver. 4). Tokyo: Biogen Japan Ltd; 2016 (in Japanese).

11. Bloomgren G, Richman S, Hotermans C, et al. Risk of natalizumab-associated progressive multifocal leukoencephalopathy. $\mathrm{N}$ Engl J Med. 2012;366:1870-80.
12. Ebers GC. Environmental factors and multiple sclerosis. Lancet Neurol. 2008;7:268-77.

13. Saida T, Kira J, Kishida S, et al. Efficacy, safety, and pharmacokinetics of natalizumab in Japanese multiple sclerosis patients: a double-blind, randomized controlled trial and open-label pharmacokinetic study. Mult Scler Relat Disord. 2017;11:25-31.

14. Saida T, Kira J-I, Kishida S, et al. Safety and efficacy of natalizumab in Japanese patients with relapsing-remitting multiple sclerosis: open-label extension study of a phase 2 trial. Neurol Ther. 2017;6:39-55.

15. Yoshimura S, Isobe N, Yonekawa T, et al. Genetic and infectious profiles of Japanese multiple sclerosis patients. PLoS One. 2012;7:e48592.

16. McElroy JP, Isobe N, Gourraud PA, et al. SNP-based analysis of the HLA locus in Japanese multiple sclerosis patients. Genes Immun. 2011;12:523-30.

17. Ochi H, Fujihara K. Demyelinating diseases in Asia. Curr Opin Neurol. 2016;29:222-8.

18. Sato DK, Callegaro D, Lana-Peixoto MA, et al. Distinction between MOG antibody-positive and AQP4 antibody-positive NMO spectrum disorders. Neurology. 2014;82:474-81.

19. Kleiter I, Hellwig K, Berthele A, et al. Failure of natalizumab to prevent relapses in neuromyelitis optica. Arch Neurol. 2012;69:239-45.

20. Phillips JT, O'Connor PW, Havrdova E, et al. Infusion-related hypersensitivity reactions during natalizumab treatment. Neurology. 2006;67:1717-8.

21. O'Connor PW, Goodman A, Kappos L, et al. Disease activity return during natalizumab treatment interruption in patients with multiple sclerosis. Neurology. 2011;76:1858-65.

22. Clerico M, Schiavetti I, De Mercanti SF, et al. Treatment of relapsing-remitting multiple sclerosis after 24 doses of natalizumab: evidence from an Italian spontaneous, prospective, and observational study (the TY-STOP Study). JAMA Neurol. 2014;71:954-60.

23. Plavina $T$, Subramanyam M, Bloomgren $G$, et al. Anti-JC virus antibody levels in serum or plasma further define risk of natalizumab-associated progressive multifocal leukoencephalopathy. Ann Neurol. 2014;76:802-12.

24. Olsson T, Achiron A, Alfredsson L, et al. Anti-JC virus antibody prevalence in a multinational multiple sclerosis cohort. Mult Scler. 2013;19:1533-8. 